\title{
Supporting Information: Characterization of hydrocarbon groups in complex mixtures using gas chromatography with unit-mass resolution electron ionization mass spectrometry
}

Gabriel Isaacman-VanWertz*, Xin Lu, Ellen Weiner, Elizabeth Smiley, Mark Widdowson

The Charles E. Via, Jr. Department of Civil and Environmental Engineering, Virginia Tech, Blacksburg, Virginia, 24061-0105, United States

Corresponding Author e-mail: ivw@vt.edu

\section{Contents}

Description of accompanying Supplementary Data . .52

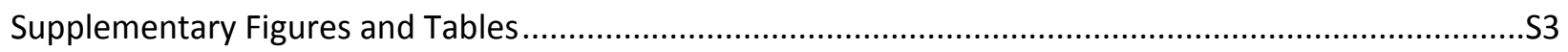

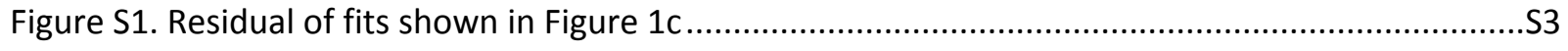

Figure S2. Comparison of observed spectra to library spectra for n-alkanes ......................................S4

Figure S3. Residuals of recreated TICS shown in Figure 2 .............................................................5

Table S1. Molecular ion intensities and retention times for all hydrocarbon groups ..........................S6 


\section{Description of accompanying Supplementary Data}

In addition to the supplementary information provide herein, Supplemental Data is provided consisting of a Microsoft Excel file containing 4 sheets. Sheet 1, "Bin Information" is a reproduction of Table S1 to allow easy access to these data. Sheets 2 through 4 contained average mass spectra calculated from all hydrocarbon mass spectra available in the library meeting the criteria $N_{C}<=30$, with no limit on $N_{D B E}$. Sheet 2, "Library spectra, Raw", contains raw average mass spectra for each compound directly as calculated from library spectra (i.e., no mathematical adjustments or transformations). Sheet 3, "Library spectra, f_Mlib_est" contains average mass spectra adjusted such that the molecular ion intensity reflects the exponential fit estimation demonstrated in Figure 1. Sheet 4, "Library spectra, f_Mlib_est_adj" contains average mass spectra adjusted such that the molecular ion intensity reflects the exponential fit estimation demonstrated in Figure 1 adjusted for the observed difference in molecular ion intensities between this GC/MS and library spectra (i.e., multiplied by 1.8). In other words, Sheet 4 represents the spectra used in this work.

In the spectra provided in Sheets 3 and 4, the relative ratios of all non-molecular ions are held the same, except for modifications in the molecular ion intensity, and each spectrum sums to unity. 

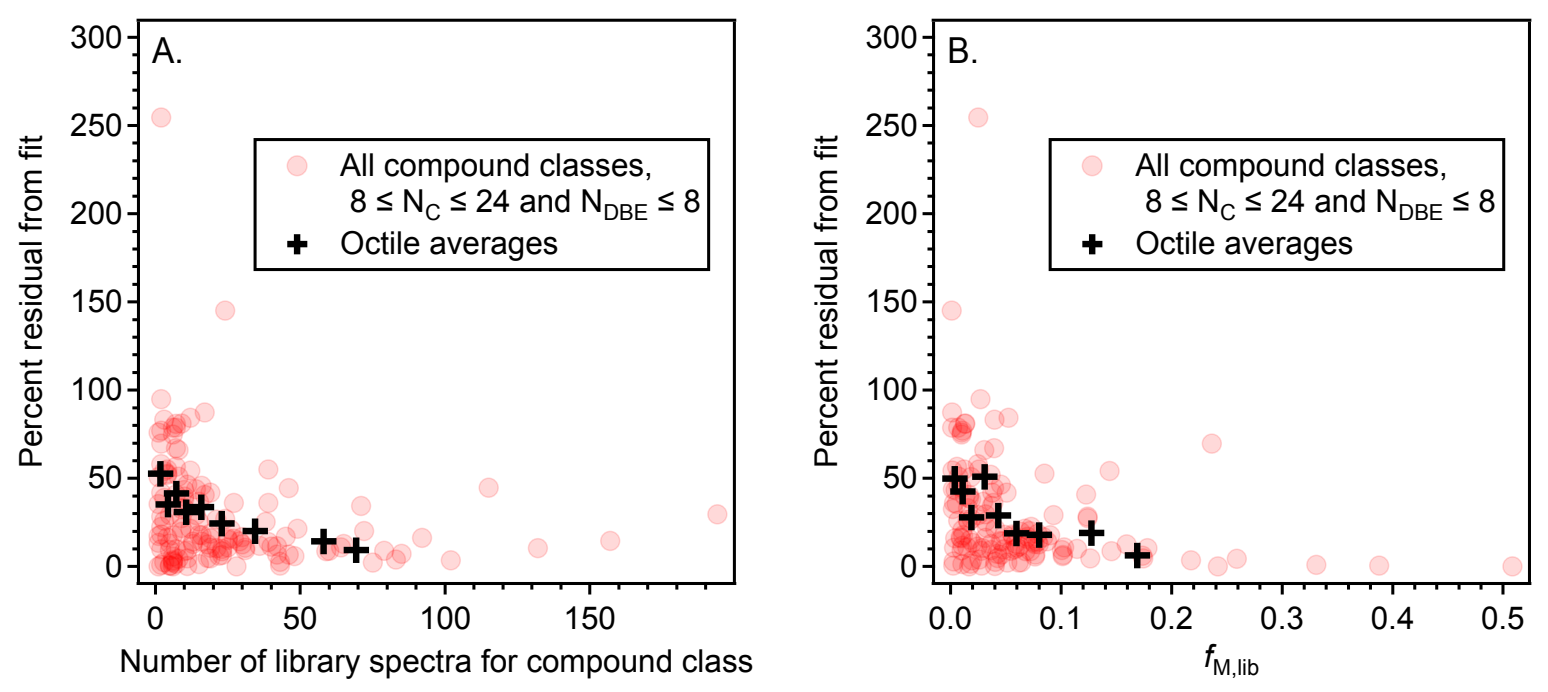

Figure S1. Residual of fits shown in Figure 1c. Percent difference between molecular ion intensity of average library mass spectra $\left(f_{M, \text { lib }}\right)$ and that estimated from an exponential fit assuming monotonic decrease as a function of $N_{C}\left(f_{M \text {,lib_est }}\right)$ as described in the text, calculated as $100 *\left(f_{M \text {,lib_est }}-f_{M, \text { lib }}\right) / f_{M \text {,lib. }}$. Shown as a function of (a) number of spectra in the library available to calculate the average spectra, and (b) molecular ion intensity of average library mass spectra. Fit residual is observed to increase as number of spectra decreases and molecular ion intensity decreases. Data shown for the range of hydrocarbon groups for which retention time windows could be determine from diesel fuel $\left(8<=N_{C}<=24\right.$, and $N_{D B E}<=8$, those provided in Table S1). Data for all hydrocarbon groups plotted as translucent red circles, with black crosses representing octiles (eight equal-sized bins of data sorted by number of spectra) 

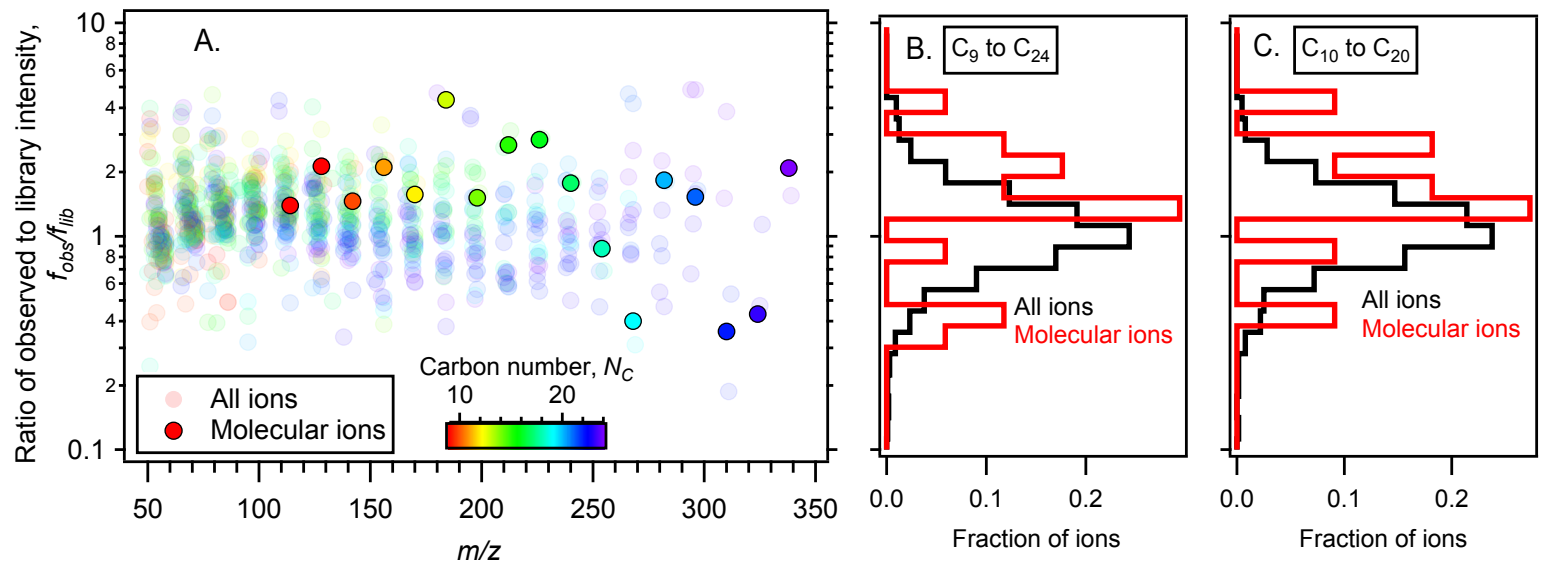

Figure S2. Comparison of observed spectra to library spectra for n-alkanes in the diesel fuel range $\left(C_{9}\right.$ to $\mathrm{C}_{24}$ ). (a) Ratio of observed ion intensity to ion intensity in the library spectra for all ions in each alkane mass spectrum, colored by $N_{c}$ of the alkane, with molecular ions of each alkane highlighted by outlines and darker colors. Frequency distributions of this ratio for (b) all alkanes in this range and (c) alkanes in the region of peak diesel signal (approximately $C_{10}$ to $C_{20}$ ). Distributions for all ions (black) are normally distributed around unity as expected, while distributions of molecular ions (red) show higher intensities in observed data than library data (by a factor of 1.8); the difference between these distributions is statistically significant for both sets on alkanes at the level of $p<0.01$ (calculated using a WilcoxonMann-Whitney test). 

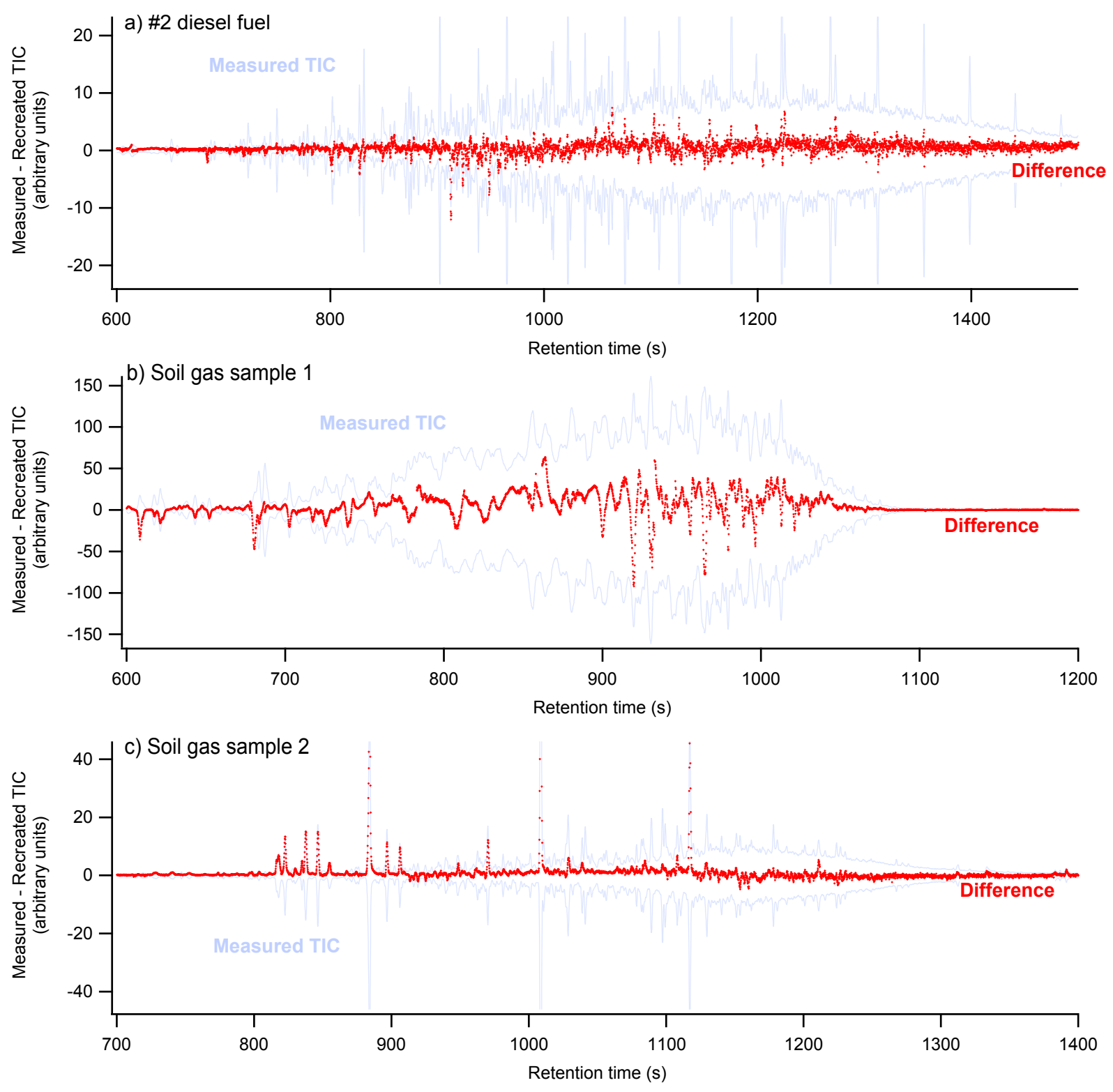

Figure S3. Residuals of recreated TICs shown in Figure 2. Absolute difference between measured and recreated TIC in red, measured TIC shown in blue as both positive and negative envelopes for context. Retention time windows are the same as shown in Figure 2. Magnitude of $y$-axis is the same as Figure 2 for panel (b), and half that of Figure 2 for panels (a) and (c) to decrease the visual influence of large resolved peaks. 
Table S1. Molecular ion intensities and retention times for all hydrocarbon groups, listed by carbon number, $N_{C}$, number of rings and double bonds, $N_{D B E}$, and molecular mass, $M$ (in units of Da). Only hydrocarbon groups with significant signal in diesel fuel are available. Fraction of the mass spectrum comprised of the molecular ion is given for the average mass spectrum calculated from the library $\left(f_{M \text {,iib }}\right)$, the estimate based on exponential fitting $\left(f_{M, \text { lib_est }}\right)$, and this estimate adjusted for differences in fragmentation between the research GC/MS and library spectra $\left(f_{M}=f_{M \text {,lib_est }} * 1.8\right)$. Retention time windows of each hydrocarbon group are provided in units of alkane-based retention index similar to Kovats index (calculated here from a polynomial fit to retention times of normal alkanes). Information for normal alkanes is separated from all other hydrocarbons.

\begin{tabular}{|c|c|c|c|c|c|c|c|}
\hline$N_{c}$ & $N_{D B E}$ & $M$ & $f_{M, l i b}$ & $f_{M, \text { lib_est }}$ & $f_{M}$ & Index Start & Index Stop \\
\hline \multicolumn{8}{|c|}{ Normal alkanes only } \\
\hline 7 & 0 & 100 & 0.02491 & 0.01655 & 0.02979 & 690 & 710 \\
\hline 8 & 0 & 114 & 0.01568 & 0.01522 & 0.02740 & 790 & 810 \\
\hline 9 & 0 & 128 & 0.01110 & 0.01403 & 0.02525 & 890 & 910 \\
\hline 10 & 0 & 142 & 0.01335 & 0.01296 & 0.02333 & 990 & 1010 \\
\hline 11 & 0 & 156 & 0.00880 & 0.01201 & 0.02161 & 1090 & 1110 \\
\hline 12 & 0 & 170 & 0.01158 & 0.01115 & 0.02007 & 1190 & 1210 \\
\hline 13 & 0 & 184 & 0.00344 & 0.01038 & 0.01869 & 1290 & 1310 \\
\hline 14 & 0 & 198 & 0.01056 & 0.00970 & 0.01745 & 1390 & 1410 \\
\hline 15 & 0 & 212 & 0.00522 & 0.00908 & 0.01634 & 1490 & 1510 \\
\hline 16 & 0 & 226 & 0.00443 & 0.00853 & 0.01535 & 1590 & 1610 \\
\hline 17 & 0 & 240 & 0.00832 & 0.00803 & 0.01446 & 1690 & 1710 \\
\hline 18 & 0 & 254 & 0.01609 & 0.00759 & 0.01367 & 1790 & 1810 \\
\hline 19 & 0 & 268 & 0.03521 & 0.00720 & 0.01295 & 1890 & 1910 \\
\hline 20 & 0 & 282 & 0.00624 & 0.00684 & 0.01231 & 1990 & 2010 \\
\hline 21 & 0 & 296 & 0.00740 & 0.00652 & 0.01174 & 2090 & 2110 \\
\hline 22 & 0 & 310 & 0.02787 & 0.00624 & 0.01123 & 2190 & 2210 \\
\hline 23 & 0 & 324 & 0.02226 & 0.00598 & 0.01077 & 2290 & 2310 \\
\hline 24 & 0 & 338 & 0.00435 & 0.00575 & 0.01036 & 2390 & 2410 \\
\hline
\end{tabular}

\section{All other hydrocarbons}

$\begin{array}{cccccccc}7 & 0 & 100 & 0.00693 & 0.00754 & 0.01358 & 671 & 690 \\ 7 & 1 & 98 & 0.04394 & 0.04617 & 0.08311 & 671 & 731 \\ 8 & 0 & 114 & 0.00719 & 0.00535 & 0.00964 & 759 & 790 \\ 8 & 1 & 112 & 0.03701 & 0.03358 & 0.06044 & 769 & 845 \\ 8 & 2 & 110 & 0.03851 & 0.04194 & 0.07549 & 872 & 879 \\ 8 & 4 & 106 & 0.07776 & 0.09801 & 0.17642 & 861 & 915 \\ 9 & 0 & 128 & 0.00302 & 0.00412 & 0.00741 & 805 & 890 \\ 9 & 1 & 126 & 0.02788 & 0.02540 & 0.04573 & 840 & 949 \\ 9 & 2 & 124 & 0.03138 & 0.03650 & 0.06570 & 927 & 1011 \\ 9 & 4 & 120 & 0.07687 & 0.07163 & 0.12894 & 933 & 1048 \\ 10 & 0 & 142 & 0.00407 & 0.00342 & 0.00615 & 917 & 990 \\ 10 & 1 & 140 & 0.01942 & 0.02010 & 0.03618 & 931 & 1053 \\ 10 & 2 & 138 & 0.02884 & 0.03184 & 0.05731 & 987 & 1066\end{array}$




\begin{tabular}{|c|c|c|c|c|c|c|c|}
\hline 10 & 3 & 136 & 0.04534 & 0.03878 & 0.06980 & 921 & 1169 \\
\hline 10 & 4 & 134 & 0.07177 & 0.05727 & 0.10308 & 1022 & 1178 \\
\hline 10 & 5 & 132 & 0.10150 & 0.09559 & 0.17206 & 1097 & 1192 \\
\hline 10 & 7 & 128 & 0.38769 & 0.38961 & 0.70130 & 1204 & 1213 \\
\hline 11 & 0 & 156 & 0.00210 & 0.00302 & 0.00544 & 1026 & 1090 \\
\hline 11 & 1 & 154 & 0.01502 & 0.01666 & 0.02998 & 1048 & 1158 \\
\hline 11 & 2 & 152 & 0.02798 & 0.02785 & 0.05014 & 1076 & 1230 \\
\hline 11 & 4 & 148 & 0.05434 & 0.04944 & 0.08899 & 1115 & 1233 \\
\hline 11 & 5 & 146 & 0.06800 & 0.07390 & 0.13303 & 1199 & 1320 \\
\hline 11 & 7 & 142 & 0.24193 & 0.24215 & 0.43588 & 1312 & 1347 \\
\hline 12 & 0 & 170 & 0.00313 & 0.00280 & 0.00504 & 1062 & 1190 \\
\hline 12 & 1 & 168 & 0.00997 & 0.01442 & 0.02596 & 1120 & 1265 \\
\hline 12 & 2 & 166 & 0.03719 & 0.02444 & 0.04399 & 1166 & 1315 \\
\hline 12 & 4 & 162 & 0.04212 & 0.04518 & 0.08132 & 1166 & 1333 \\
\hline 12 & 5 & 160 & 0.06176 & 0.06316 & 0.11368 & 1213 & 1483 \\
\hline 12 & 7 & 156 & 0.17384 & 0.16313 & 0.29364 & 1416 & 1503 \\
\hline 13 & 0 & 184 & 0.00109 & 0.00267 & 0.00481 & 1181 & 1290 \\
\hline 13 & 1 & 182 & 0.01074 & 0.01297 & 0.02335 & 1231 & 1382 \\
\hline 13 & 2 & 180 & 0.02429 & 0.02152 & 0.03874 & 1213 & 1386 \\
\hline 13 & 4 & 176 & 0.04596 & 0.04286 & 0.07714 & 1279 & 1425 \\
\hline 13 & 5 & 174 & 0.06950 & 0.05783 & 0.10409 & 1298 & 1523 \\
\hline 13 & 6 & 172 & 0.08728 & 0.07474 & 0.13453 & 1444 & 1628 \\
\hline 13 & 7 & 170 & 0.12653 & 0.12079 & 0.21742 & 1503 & 1617 \\
\hline 13 & 8 & 168 & 0.17437 & 0.16632 & 0.29938 & 1513 & 1554 \\
\hline 14 & 0 & 198 & 0.00169 & 0.00260 & 0.00468 & 1263 & 1390 \\
\hline 14 & 1 & 196 & 0.01077 & 0.01203 & 0.02166 & 1292 & 1479 \\
\hline 14 & 2 & 194 & 0.01227 & 0.01902 & 0.03424 & 1298 & 1523 \\
\hline 14 & 3 & 192 & 0.03927 & 0.02179 & 0.03922 & 1345 & 1564 \\
\hline 14 & 4 & 190 & 0.04054 & 0.04159 & 0.07487 & 1333 & 1503 \\
\hline 14 & 5 & 188 & 0.06229 & 0.05518 & 0.09933 & 1369 & 1649 \\
\hline 14 & 6 & 186 & 0.07995 & 0.06988 & 0.12578 & 1483 & 1766 \\
\hline 14 & 7 & 184 & 0.08402 & 0.09809 & 0.17657 & 1564 & 1785 \\
\hline 14 & 8 & 182 & 0.14560 & 0.13287 & 0.23916 & 1437 & 1907 \\
\hline 15 & 0 & 212 & 0.00193 & 0.00256 & 0.00461 & 1386 & 1490 \\
\hline 15 & 1 & 210 & 0.01843 & 0.01142 & 0.02056 & 1369 & 1594 \\
\hline 15 & 2 & 208 & 0.01686 & 0.01688 & 0.03039 & 1406 & 1606 \\
\hline 15 & 3 & 206 & 0.01621 & 0.02031 & 0.03656 & 1479 & 1649 \\
\hline 15 & 4 & 204 & 0.03157 & 0.04090 & 0.07363 & 1369 & 1649 \\
\hline 15 & 5 & 202 & 0.06028 & 0.05388 & 0.09698 & 1444 & 1693 \\
\hline 15 & 6 & 200 & 0.07873 & 0.06765 & 0.12177 & 1523 & 1832 \\
\hline 15 & 7 & 198 & 0.07618 & 0.08593 & 0.15468 & 1649 & 1832 \\
\hline 15 & 8 & 196 & 0.10267 & 0.11382 & 0.20487 & 1479 & 2140 \\
\hline 16 & 0 & 226 & 0.00136 & 0.00254 & 0.00457 & 1444 & 1590 \\
\hline 16 & 1 & 224 & 0.00950 & 0.01102 & 0.01984 & 1473 & 1700 \\
\hline
\end{tabular}




\begin{tabular}{|c|c|c|c|c|c|c|c|}
\hline 16 & 2 & 222 & 0.01776 & 0.01505 & 0.02710 & 1473 & 1693 \\
\hline 16 & 3 & 220 & 0.01526 & 0.01930 & 0.03475 & 1523 & 1785 \\
\hline 16 & 4 & 218 & 0.04256 & 0.04053 & 0.07295 & 1444 & 1738 \\
\hline 16 & 5 & 216 & 0.03752 & 0.05323 & 0.09581 & 1523 & 1832 \\
\hline 16 & 6 & 214 & 0.04553 & 0.06663 & 0.11994 & 1606 & 1982 \\
\hline 16 & 7 & 212 & 0.06852 & 0.07942 & 0.14295 & 1738 & 1932 \\
\hline 16 & 8 & 210 & 0.11437 & 0.10297 & 0.18534 & 1594 & 2195 \\
\hline 17 & 0 & 240 & 0.00260 & 0.00253 & 0.00455 & 1523 & 1690 \\
\hline 17 & 1 & 238 & 0.00986 & 0.01077 & 0.01938 & 1503 & 1782 \\
\hline 17 & 2 & 236 & 0.01145 & 0.01349 & 0.02427 & 1523 & 1785 \\
\hline 17 & 3 & 234 & 0.01052 & 0.01862 & 0.03352 & 1585 & 1858 \\
\hline 17 & 4 & 232 & 0.04023 & 0.04032 & 0.07258 & 1483 & 1832 \\
\hline 17 & 5 & 230 & 0.04815 & 0.05290 & 0.09523 & 1606 & 1932 \\
\hline 17 & 6 & 228 & 0.05854 & 0.06617 & 0.11910 & 1649 & 2034 \\
\hline 17 & 7 & 226 & 0.06341 & 0.07592 & 0.13666 & 1693 & 2086 \\
\hline 17 & 8 & 224 & 0.05251 & 0.09679 & 0.17422 & 1700 & 2251 \\
\hline 18 & 0 & 254 & 0.00580 & 0.00252 & 0.00453 & 1641 & 1790 \\
\hline 18 & 1 & 252 & 0.00727 & 0.01060 & 0.01908 & 1606 & 1895 \\
\hline 18 & 2 & 250 & 0.00680 & 0.01214 & 0.02186 & 1628 & 1932 \\
\hline 18 & 3 & 248 & 0.01300 & 0.01816 & 0.03268 & 1649 & 1932 \\
\hline 18 & 4 & 246 & 0.03681 & 0.04021 & 0.07238 & 1564 & 1932 \\
\hline 18 & 5 & 244 & 0.04621 & 0.05274 & 0.09494 & 1738 & 1982 \\
\hline 18 & 6 & 242 & 0.09315 & 0.06596 & 0.11872 & 1738 & 2140 \\
\hline 18 & 7 & 240 & 0.09007 & 0.07405 & 0.13330 & 1882 & 2140 \\
\hline 18 & 8 & 238 & 0.07867 & 0.09327 & 0.16788 & 1782 & 2306 \\
\hline 19 & 0 & 268 & 0.00444 & 0.00251 & 0.00452 & 1709 & 1890 \\
\hline 19 & 1 & 266 & 0.01566 & 0.01049 & 0.01889 & 1716 & 2008 \\
\hline 19 & 2 & 264 & 0.00729 & 0.01100 & 0.01980 & 1738 & 2034 \\
\hline 19 & 3 & 262 & 0.01812 & 0.01784 & 0.03211 & 1738 & 2060 \\
\hline 19 & 4 & 260 & 0.04890 & 0.04015 & 0.07227 & 1738 & 2113 \\
\hline 19 & 5 & 258 & 0.06708 & 0.05267 & 0.09480 & 1738 & 2086 \\
\hline 19 & 6 & 256 & 0.07222 & 0.06586 & 0.11854 & 1761 & 2251 \\
\hline 19 & 7 & 254 & 0.03985 & 0.07305 & 0.13149 & 1832 & 2195 \\
\hline 19 & 8 & 252 & 0.10063 & 0.09126 & 0.16427 & 1895 & 2451 \\
\hline 20 & 0 & 282 & 0.00140 & 0.00251 & 0.00452 & 1714 & 1990 \\
\hline 20 & 1 & 280 & 0.01057 & 0.01042 & 0.01876 & 1785 & 2100 \\
\hline 20 & 2 & 278 & 0.01685 & 0.01001 & 0.01803 & 1761 & 2140 \\
\hline 20 & 3 & 276 & 0.02489 & 0.01763 & 0.03173 & 1761 & 2168 \\
\hline 20 & 4 & 274 & 0.03194 & 0.04012 & 0.07221 & 1785 & 2195 \\
\hline 20 & 5 & 272 & 0.03869 & 0.05263 & 0.09473 & 1832 & 2195 \\
\hline 20 & 6 & 270 & 0.03938 & 0.06581 & 0.11846 & 1789 & 2309 \\
\hline 20 & 7 & 268 & 0.12263 & 0.07251 & 0.13052 & 1836 & 2364 \\
\hline 20 & 8 & 266 & 0.12415 & 0.09012 & 0.16222 & 2008 & 2479 \\
\hline 21 & 0 & 296 & 0.00249 & 0.00251 & 0.00452 & 1885 & 2090 \\
\hline
\end{tabular}




\begin{tabular}{llllllll}
21 & 1 & 294 & 0.02478 & 0.01038 & 0.01868 & 1813 & 2140 \\
21 & 2 & 292 & 0.01858 & 0.00917 & 0.01651 & 1836 & 2306 \\
21 & 3 & 290 & 0.00061 & 0.01748 & 0.03146 & 1875 & 2335 \\
21 & 4 & 288 & 0.02932 & 0.04010 & 0.07218 & 1836 & 2364 \\
21 & 5 & 286 & 0.05145 & 0.05261 & 0.09469 & 1836 & 2364 \\
21 & 6 & 284 & 0.06421 & 0.06579 & 0.11843 & 1885 & 2364 \\
21 & 7 & 282 & 0.07651 & 0.07222 & 0.13000 & 1885 & 2364 \\
21 & 8 & 280 & 0.07301 & 0.08947 & 0.16105 & 2100 & 2479 \\
22 & 0 & 310 & 0.00994 & 0.00251 & 0.00452 & 1974 & 2190 \\
22 & 1 & 308 & 0.01695 & 0.01035 & 0.01862 & 1907 & 2251 \\
22 & 2 & 306 & 0.00671 & 0.00845 & 0.01522 & 1907 & 2451 \\
22 & 3 & 304 & 0.03619 & 0.01738 & 0.03128 & 1907 & 2451 \\
22 & 4 & 302 & 0.03289 & 0.04009 & 0.07216 & 1957 & 2451 \\
22 & 5 & 300 & 0.05829 & 0.05260 & 0.09468 & 1982 & 2451 \\
22 & 6 & 298 & 0.14380 & 0.06578 & 0.11841 & 1982 & 2451 \\
22 & 7 & 296 & 0.05080 & 0.07207 & 0.12973 & 1982 & 2451 \\
22 & 8 & 294 & 0.12412 & 0.08910 & 0.16038 & 2140 & 2594 \\
23 & 0 & 324 & 0.01315 & 0.00251 & 0.00452 & 2008 & 2290 \\
23 & 1 & 322 & 0.03046 & 0.01033 & 0.01859 & 2008 & 2364 \\
23 & 3 & 318 & 0.00984 & 0.01731 & 0.03116 & 1982 & 2537 \\
23 & 4 & 316 & 0.02586 & 0.04009 & 0.07215 & 1982 & 2537 \\
23 & 6 & 312 & 0.05611 & 0.06578 & 0.11840 & 1982 & 2537 \\
23 & 8 & 308 & 0.00387 & 0.08889 & 0.16000 & 2251 & 2623 \\
24 & 0 & 338 & 0.01326 & 0.00251 & 0.00452 & 2060 & 2390 \\
24 & 1 & 336 & 0.00885 & 0.01031 & 0.01857 & 2113 & 2479 \\
24 & 2 & 334 & 0.00540 & 0.00731 & 0.01316 & 2060 & 2537 \\
24 & 3 & 332 & 0.01995 & 0.01727 & 0.03108 & 2060 & 2594 \\
24 & 4 & 330 & 0.08480 & 0.04008 & 0.07215 & 2060 & 2594 \\
24 & 5 & 328 & 0.02697 & 0.05259 & 0.09466 & 2060 & 2594 \\
\hline & 6 & 326 & 0.05570 & 0.06578 & 0.11840 & 2060 & 2594 \\
\hline 24 & 324 & 0.23648 & 0.07194 & 0.12950 & 2060 & 2594 \\
\hline 24 & 322 & 0.02505 & 0.08877 & 0.15979 & 2364 & 2737
\end{tabular}

